\title{
Bi-Virus SIS Epidemics over Networks: Qualitative Analysis
}

\author{
Augusto Santos*, José M. F. Moura ${ }^{\natural}$, and João M. F. Xavier ${ }^{\dagger}$
}

\begin{abstract}
The paper studies the qualitative behavior of a set of Ordinary Differential Equations that models the dynamics of bi-virus epidemics over bilayer networks. Each layer is a weighted digraph associated with a strain of virus; the weights $\gamma_{i j}^{z}$ represent the rates of infection from node $i$ to node $j$ of strain $z$. We establish a sufficient condition on the $\gamma$ 's that guarantees survival of the fittest-only one strain survives. We propose an ordering of the weighted digraphs, the $\star$-order, and show that if the weighted digraph of strain $y$ is $\star$-dominated by the weighted digraph of strain $x$, then $y$ dies out in the long run. We prove that the orbits of the ODE accumulate to an attractor. Due to the coupled nonlinear high-dimension nature of the ODEs, there is no natural Lyapunov function to study their global qualitative behavior. We prove our results by combining two important properties of these ODEs: (i) monotonicity under a partial ordering on the set of graphs; and (ii) dimension-reduction under symmetry of the graphs. Property (ii) allows us to fully address the survival of the fittest for regular graphs. Then, by bounding the epidemics dynamics for generic networks by the dynamics on regular networks, we prove the result for general networks.
\end{abstract}

Keywords: Bi-virus epidemics, Survival of the fittest, Differential equations, Qualitative analysis, Attractor, Basin of attraction.

* A. Santos is with the Dep. of Electrical and Computer Engineering, Carnegie Mellon University, USA, and Instituto de Sistemas e Robotica (ISR), Instituto Superior Técnico (IST), Av. Rovisco Pais, Lisboa, Portugal (augustos@andrew.cmu.edu).

J. M. F. Moura is with the Dep. of Electrical and Computer Engineering, Carnegie Mellon University, Pittsburgh, PA 15213, USA, ph:(412)268-6341, fax: (412)268-3890 (moura@ece.cmu.edu).

$\dagger$ J. M. F. Xavier is with ISR, IST, Av. Rovisco Pais, Lisboa, Portugal (jxavier@isr.ist.utl.pt).

The work of J. M. F. Moura and A. Santos was supported in part by the National Science Foundation under Grant \# CCF-1011903 and in part by the Air Force Office of Scientific Research under Grant \# FA-95501010291.

The work of J. M. F. Xavier and A. Santos was supported by the Fundação para a Ciência e a Tecnologia (FCT) through the Carnegie Mellon|Portugal Program under Grant SFRH/BD/33516/2008, CMU-PT/SIA/0026/2009, SFRH/BD/33518/2008, PTDC/EMS$\mathrm{CRO} / 2042 / 2012$, and by ISR/IST pluriannual funding (POSC program, FEDER).

\section{INTRODUCTION AND BRIEF REVIEW OF THE LITERATURE}

Historical remarks. The dynamics of physical systems are often modeled by ordinary differential or difference equations (ODEs). Mathematical models for epidemics have a long history starting possibly with the work of Daniel Bernoulli in the mid-eighteen century, [1], defending inoculation against smallpox. In the first three decades of the twentieth century, among others, the works of Hamer [2] and Kermack and McKendrick [3] laid the foundations of mathematical epidemiology. The population is divided in compartments, e.g., susceptibles, infectious, exposed, removed, and the epidemics are modeled by ODEs governing the rates of flow between these compartments, see [4] for a comprehensive collection of such models. These models have been established for many infectious diseases from the historical plagues affecting millions over the centuries, to outbreaks of cholera, malaria, influenza, or HIV.

ODE model motivation. Such logistic-like ODE models can be motivated through mean-field approximation arguments, e.g., [5], [6]. More formally, in [7], we proved that the solutions to the Susceptible-InfectedSusceptible (SIS) bi-virus ODEs (4)-(5) represent the exact weak limit (under an appropriate topology on the set of sample paths) of a stochastic process representing the fraction of infected nodes at each partite on a completemultipartite network and evolving according to a peerto-peer stochastic model of infection (Harris contact process). Namely, we proved that, as the number of nodes at each partite grows to infinite (in a controlled way), the vector collecting the fraction of infected nodes at each partite converges weakly to the solution of the ODE. For the sake of completeness, we remark that multipartite structures find motivation in real life epidemics. For example, the dengue disease is spread between humans and Aedes Aegipty mosquitoes according to a bipartite structure, refer to [8]. Indeed, a healthy human gets infected once bitten by an infected mosquito. A healthy mosquito gets infected once it bites an infected human being. The disease does not spread among humans nor does it spread among mosquitoes, i.e., only cross-species infection is allowed. Therefore, the underlying network of infections has two partites connected together: one 
comprised of mosquitoes and the other of human beings. Therefore, such ODEs conform to a natural model to study the evolution of the fraction of infected nodes at each partite on a large-scale complete-multipartite network (from [7]) as in Dengue-like epidemics, or to study the evolution of the likelihood of infection of nodes in an arbitrary network as, e.g., in [5], [6] for singlevirus, or [9] for bi-virus.

Equilibria versus qualitative analysis in the literature. There is a vast body of literature dedicated to the study of single-virus epidemics over networks. References [6], [10] provide a comprehensive review of the epidemics literature. All these references focus mostly on studying the equilibria of imposed idealized differential equation models with a few providing heuristics on the transitory behavior of such dynamical systems. Reference [5] provides several bounds on the equilibria of a single virus epidemics. These references concentrate on studying the equilibria of such ODE models, i.e., determining the equilibria, or properties of the equilibria, or the local (often linearized) dynamics about the equilibria. They do not study rigorously their qualitative behavior, i.e., do not characterize the attractors and basins of attraction of the dynamical system. Reference [6] also attempts to briefly study the qualitative behavior of some epidemics models resorting to heuristic arguments, e.g., linearizing the ODEs when the degree of infection is small. We do not evoke such approximations in this work. We remark that the discretetime counter-part of such ODEs present chaotic behavior and do not, in general, converge to the equilibria (refer to the logistic map, e.g., in [11]), and thus, results on the global stability of the system are crucial to understand the behavior of its solutions from the initial conditions to the attractors. Reference [12] does provide a rigorous qualitative analysis for the single virus epidemics dynamics - equation (3) - based on Lyapunov stability theory. As we explain in Section III, the results in [12] for the single virus dynamics do not apply to the bivirus case, and we need to develop a new methodology. Reference [13] studies via numerical simulations the epidemics over big cities resorting to dynamical systems similar to the one in equation (3), to model the mixing of different interacting groups of individuals.

Bi-virus motivation and some references. Bi-virus dynamics is motivated by the virus pandemic vs vaccination problem. In many real life settings, once infected, an individual cannot be vaccinated; and, if vaccinated, it is not prone (ideally) to be infected. This partitions the population in disjoint sets: infected; not infected and not vaccinated (susceptible); and vaccinated (and thus, not susceptible). This exclusion principle couples the dynamics of spreading of the virus with the dynamics of vaccines: one cannot understand the bi-virus dynamics of virus-vaccines (ODE (4)-(5)) by studying it separately (e.g., via the single-virus ODE (3)). While the virus spreads across the population according to the underlying network of contacts, [14], [15] suggest that vaccines obey similar dynamics: the social network of influences plays a major role on the decision of parents vaccinating their children. Also, recent research results in malaria vaccines (e.g., [16]) suggest that infection inhibition via mosquitoes bites is possible. In other words, the vaccines would spread across the population via similar dynamics as the main virus ${ }^{1}$. In this case, one recovers the bivirus epidemics setting in the network where the vaccine may be cast as another virus strain spreading similarly to the main virus with mutual inhibition between the strains. Therefore, the bi-virus dynamics, see (4)-(5) below, are a natural model to study the evolution of infected and vaccinated individuals. The analytic study of this dynamical system can inform the regulatory policies of vaccination to preventing the persistence of the epidemics. Similar models for bi-virus competition in a network are addressed in references [9], [17], [18]. The common feature among these papers and the majority of the literature in epidemics is that they perform local analysis - i.e., determine properties of the equilibria, or study the dynamical system on small neighborhoods of the equilibrium points. In contrast, we study our dynamical system via global stability analysis.

Main contribution of this paper. This paper focus on the qualitative analysis of the bi-virus coupled nonlinear ODEs dynamics (4)-(5). We do not focus on characterizing purely the equilibria (or properties of the equilibria); we devise tools that guarantee to where the orbits of the dynamical system accumulate to. More precisely, we prove that, under some condition on the rate parameters of the ODE (4)-(5), the orbits accumulate to an attractor $\mathcal{A}$ that we characterize. This is further interpreted as a natural selection phenomenon taking course in the long run: the weaker strain dies out whereas the stronger strain survives with a degree of infection above a threshold that we determine. Due to the high-dimension and nonlinearity of the ODEs, the bi-virus epidemics defies the use of Lyapunov methods. Our approach explores three properties of the ODE (4)(5) to establish the sufficient condition for survival of the fittest: (i) monotonicity under initial conditions; (ii) monotonicity under an appropriate partial ordering on the set of graphs; and (iii) complete qualitative characterization of (4)-(5) for regular networks. By combining these three properties and introducing a new ordering on the set of weighted digraphs (referred to as $\star$-order), we can obtain a sufficient condition for survival of the fittest in

\footnotetext{
${ }^{1}$ Technically, malaria is not caused by a virus, but by a protozoan. For our purposes, the relevant feature lies in the dynamics of spread and not in the nature of the viral agent.
} 
general weighted digraphs: if the strain $y$ is $\star$-dominated by the strain $x$, then $y$ dies out, i.e., it cannot co-habit the network along with the strain $x$ in the long run. Our approach is based on bounding the epidemics dynamics for generic networks by the epidemics dynamics on regular networks. More precisely, one can appropriately bound the dynamics of the strains of virus in general networks by the dynamics over inner/outer regular networks to show that the ODE over general networks exhibit natural selection, as we will make clearer below. We explore the fact that, if the underlying network is regular with all infection rates constant across edges, then the global attractor is an equilibrium point (to be proved). Remark that the sub (or inner)/supper (or outer) regular graphs of a graph $G$ always exist, even if trivially - the isolated nodes and the complete networks, respectively. Although there is no closed form for the number of regular subnetworks on $n$ nodes, see [19], [20], [21], reference [22] shows that, in general, for random networks in the asymptotic limit of large networks there is a high probability that it contains a regular non-trivial subnetwork.

What this paper is about. This paper is about a rigorous global qualitative analysis of a set of ODEs that model the evolution of two strains of virus in a network. We determine a sufficient condition on the parameters of these ODEs (a.k.a., virus rates) under which the orbits of these ODEs converge to a survival of the fittest attractor, regardless of the initial conditions.

What this paper is not about. In this paper, we do not study the original stochastic system giving rise (in the limit of large network) to the ODE (4)-(5) as established in [7]. In this paper, we do not resort to approximations about the equilibria - such as linearizing the system about the equilibria - nor do we pursue a local analysis about the equilibria.

Summary of the paper. Section II sets-up the main notation, important inequalities between networks, and the ODEs to be studied through out the paper. Section III illustrates our model analysis in the simpler case of single-virus epidemics. Section IV establishes the main result on the survival of the fittest associated with the bi-virus dynamics (4)-(5) in Theorem 20. We include some auxiliary Theorems, Theorems 22, 23, and 24, and the corresponding proofs in Section V. Such Theorems are crucial to establish the main Theorem, Theorem 20, but, for the sake of clarity, we omit them in Section IV. Concluding remarks are in Section VI.

Preliminary Notation. Symbols $\mathbb{R}_{+}$and $\mathbb{R}_{++}$represent the set of nonnegative and positive, respectively, real numbers; $\mathbb{N}=\{1,2, \ldots\}$ represents the set of natural numbers. We define $\mathbf{1}_{N} \in \mathbb{R}^{N}$ (and $\mathbf{0}_{N} \in \mathbb{R}^{N}$ ) as the vectors with all entries equal to one (respectively, 0) the subindex may be omitted whenever the dimension is clear from the context. Let $\mathbf{x}, \mathbf{y} \in \mathbb{R}^{N}$, we define $\mathbf{x} \geq \mathbf{y}$ as the pointwise inequality, i.e., $x_{i} \geq y_{i}$ for all $i=1, \ldots, N$. We refer to $\mathbf{x} \ngtr 0$ as $\mathbf{x} \notin \mathbb{R}_{++}^{N}$. Define $|V|$ as the cardinality of the set $V$. Let $x, y \in \mathbb{R}$ and define $x \wedge y$ as: $x \wedge y=x$, if $x \leq y$ or $x \wedge y=y$, if otherwise. Let $x, y \in \mathbb{R}$ and define $x \vee y$ as: $x \vee y=x$, if $x \geq y$ or $x \vee y=y$, if otherwise. We define the Hadamard pointwise product $\mathbf{x} \odot \mathbf{y}$ as

$$
\mathbf{x} \odot \mathbf{y}=\left(x_{1} y_{1}, \ldots, x_{N} y_{N}\right) .
$$

Let $\mathbf{A} \otimes \mathbf{y}$ be the Kronecker product

$$
\mathbf{A} \otimes \mathbf{y}=\left(a_{i j} \mathbf{y}\right)_{i j} .
$$

\section{Flow In NeTwORKS}

In this Section, we present the bi-virus ODE model to be studied along its associated flow, we set the main notation and introduce basic definitions to be used throughout. We present useful inequalities on networks, namely, we propose the $\star$-inequality ' $\preceq^{\star}$ ' that will be important in Section IV to draw a sufficient condition for survival of the fittest: if $G^{y} \preceq^{\star} G^{x}$, where $G^{y}, G^{x}$ are the weighted digraphs - to be defined - associated with the strains $x, y$, then the strain $y$ dies out. We start by defining some graph constructs.

Definition 1 (Graph). The ordered pair $G=(V, E)$, is a graph; where $V \subset \mathbb{N}$ and $E \subset V \times V$ are the finite set of nodes and edges, respectively.

Unless otherwise stated, $V$, with cardinality $|V|=N$, is defined by default as

$$
V=\{1, \ldots, N\} .
$$

Next, we define a weighted digraph that we associate with a virus infection: it couples the underlying graph of potential infections with the rates of infection between nodes. In order to avoid repeatimg the term "weighted digraph" recursively, we denote it simply by "e-network".

Definition 2 (E-Network). The ordered pair $G=(V, \gamma)$ is an e-network, where $V \subset \mathbb{N}$ is finite and $\gamma: V \times V \rightarrow$ $\mathbb{R}_{+}$is a nonnegative function;

$$
\mathcal{E}(V):=\left\{G=(V, \gamma): \gamma: V \times V \rightarrow \mathbb{R}_{+}\right\}
$$

is the set of e-networks with fixed node set $V$. If the particulars of $V$ are not relevant, we write $\mathcal{E}(|V|)$ instead of $\mathcal{E}(V)$.

The $N \times N$ matrix/function $\gamma$ is the infection rate of a virus between any two arbitrary nodes with $\gamma(i, j)$, or $\gamma_{i j}$, standing for the rate of infection from node $i$ towards node $j$. In Fig. 1, we depict an e-network. The path-flow graph of infection is specified by the support of $\gamma$ that we now define. 


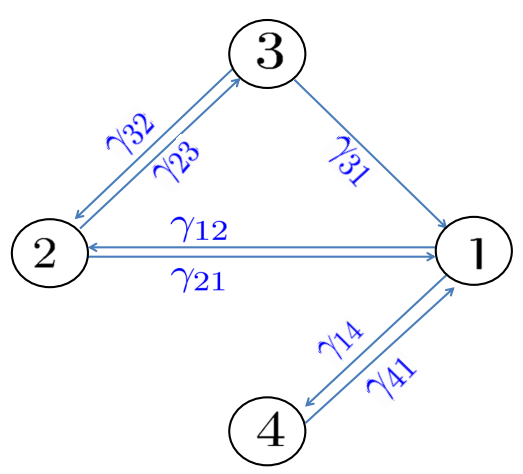

Figure 1: Representation of an e-network $G=(V, \gamma)$, with $V=\{1,2,3,4\}$. The edges associated with null rates $\gamma$ are not depicted. That is, $\gamma_{24}=\gamma_{42}=0$ and $\gamma_{13}=0$.

Definition 3 (Support Graph of an E-Network). Let $G=$ $(V, \gamma)$ be an e-network. We call

$$
\operatorname{supp}(G)=\left(V, \gamma^{-1}\left(\mathbb{R}_{++}\right)\right)
$$

the support graph of the e-network $G$, where $\gamma^{-1}\left(\mathbb{R}_{++}\right)$ is the inverse image of $\mathbb{R}_{++}$by

$$
\gamma: V \times V \rightarrow \mathbb{R}_{+}
$$

or, in words, the set of edges $(i, j)$ where $\gamma(i, j)>0$.

The support graph represents the graph where an edge from node $i$ to node $j$ is placed whenever $\gamma(i, j)>0$ and not placed if otherwise $\gamma(i, j)=0$. This provides us with the path-flow of infection of a virus associated with the e-network $G$. Thus, an e-network $G$ provides us not only with the topology of the network environment as seen by the virus, but also with the quantitative rates $\gamma(i, j)$ of infection attached to each edge $(i, j)$. We assume in this work that e-networks are the defining feature of viruses: each strain bears an underlying e-network and they are assumed different (with possibly different topologies of the corresponding support graphs). Therefore, the neighborhood of a node $a$ - set of nodes that can directly infect the node $a-$ is sensitive to the underlying strain of virus. For instance, in Fig.2, node 4 is neighbor to node 3 with respect to strain $y$, but not with respect to strain $x$. Next, we formally define the neighborhood of a node in e-networks and introduce the appropriate notation. The concept of second order or, more generally, $n$th order neighborhood is useful and will be also introduced.

Definition 4 (Neighborhood). Let $G=(V, \gamma)$ be an enetwork, and $i \in V$ be a node in $G$. Define $\mathcal{N}(i, G)$ as the 1-hop neighborhood of node $i$ in $G$ and write it as

$$
\mathcal{N}(i, G):=\left\{j \in V: j \rightarrow_{G} i\right\}
$$

where $j \rightarrow_{G} i$ means $\gamma(j, i)>0$, i.e., node $j$ can directly infect node $i$. Write $\mathcal{N}^{2}(i, G)$ as the 2 nd order neighborhood of $i$, that is,

$$
j \in \mathcal{N}^{2}(i, G)
$$

if and only if the shortest path connecting $j$ to $i$ (a.k.a. geodesic) has length 2 hops. Inductively, say

$$
j \in \mathcal{N}^{n}(i, G)
$$

if and only if there exists

$$
k \in \mathcal{N}^{n-1}(i, G)
$$

with $j \rightarrow_{G} k$, i.e., the geodesic connecting $j$ to $i$ has a length of $n$ hops. If there is no ambiguity, we will suppress $G$ and we will write $\mathcal{N}^{n}(i)$ and $i \rightarrow j$, instead.

The degree of node $i \in V$ in $G$ is

$$
d_{i}(G):=|\mathcal{N}(i, G)| \text {. }
$$

From the definition,

$$
\mathcal{N}^{l}(i) \cap \mathcal{N}^{m}(i)=\emptyset
$$

whenever $l \neq m$, i.e., $\left(\mathcal{N}^{n}(i)\right)_{n}$ partitions $V$.

We now present the single-virus epidemics ODE model and its underlying flow.

Definition 5 (Flow of Single-Virus ODE). Let $\mathcal{D}(V)=$ $[0,1]^{|V|}$. Define the function

$$
\begin{aligned}
\mathbf{y}: \mathbb{R}_{+} \times \mathcal{D}(V) \times \mathcal{E}(V) & \longrightarrow \mathcal{D}(V), \\
\left(t, \mathbf{y}_{0} ; G\right) & \longmapsto \mathbf{y}\left(t, \mathbf{y}_{0} ; G\right)
\end{aligned}
$$

where $\left(\mathbf{y}\left(t, \mathbf{y}_{0} ; G\right)\right)_{t \geq 0}$ is the solution to the ODE

$$
\dot{y}_{i}(t)=\underbrace{\left(\sum_{j \in V} \gamma(j, i) y_{j}(t)\right)\left(1-y_{i}(t)\right)-y_{i}(t)}_{F_{i}(\mathbf{y}(t) ; G)}
$$

for $i=1, \ldots, N$ for $N=|V|$, initial condition $\mathbf{y}_{0} \in$ $\mathcal{D}(V)$, and underlying e-network

$$
G=(V, \gamma) \in \mathcal{E}(V) .
$$

For fixed $G,\left(\mathbf{y}\left(t, \mathbf{y}_{0} ; G\right)\right)_{t \geq 0}$ is the flow for $t \geq 0$ of virus $y$ over the e-network $G$, starting from $\mathbf{y}_{0}$ at time $t=0$. Whenever $V$ is clear from the context, we will write simply $\mathcal{D}$ and $\mathcal{E}$.

We observe that, given an e-network $G \in \mathcal{E}(V)$, the flow $\left(\mathbf{y}\left(t, \mathbf{y}_{0} ; G\right)\right)$ to the ODE (3) is well defined - it is uniquely determined for all time $t, t \geq 0$. Indeed, the underlying vector field

$$
\mathbf{F}(\cdot, G)=\left(F_{1}(\cdot, G), \ldots, F_{N}(\cdot, G)\right)
$$

is (globally) Lipschitz over the domain $\mathcal{D}=[0,1]^{N}$ for 
all $G \in \mathcal{E}(V)$. Note that the set $\mathcal{D}$ is invariant with respect to the dynamics, that is, $\mathbf{y}\left(t, \mathbf{y}_{0} ; G\right) \in \mathcal{D}$ for all time $t$ whenever $\mathbf{y}_{0} \in \mathcal{D}$. The fact that $\mathcal{D}$ is compact further implies that the solutions are defined for all $t$, $t \geq 0$. Whenever the underlying e-network $G$ is clear from the context or previously fixed, we will write $y_{i}(t)$ or $y_{i}\left(t, \mathbf{y}_{0}\right)$ instead of $y_{i}\left(t, \mathbf{y}_{0} ; G\right)$.

Our goal in this paper is to study the bi-virus epidemics dynamics that we introduce next.

Definition 6 (Flow of bi-Virus ODE). Let

$$
\mathcal{D}_{2}(V):=\left\{(\mathbf{x}, \mathbf{y}) \in \mathcal{D}(V) \times \mathcal{D}(V): \mathbf{x}+\mathbf{y} \leq \mathbf{1}_{N}\right\},
$$

with $\mathcal{D}(V)=[0,1]^{|V|}$. Define the function

$$
\begin{aligned}
& (\mathbf{x}, \mathbf{y}): \mathbb{R}_{+} \times \mathcal{D}_{2}(V) \times \mathcal{E}^{2}(V) \longrightarrow \mathcal{D}_{2}(V) \\
& \left(t, \mathbf{z}_{0} ; G^{x, y}\right) \longmapsto\left(\mathbf{x}\left(t, \mathbf{z}_{0} ; G^{x, y}\right),\right. \\
& \left.\mathbf{y}\left(t, \mathbf{z}_{0} ; G^{x, y}\right)\right)
\end{aligned}
$$

where $\left((\mathbf{x}, \mathbf{y})\left(t, \mathbf{z}_{0} ; G^{x, y}\right)\right)_{t \geq 0}$ is the solution to the $O D E$

$$
\begin{aligned}
\dot{x}_{i}(t)= & \left(\sum_{j \in V} \gamma^{x}(j, i) x_{j}(t)\right)\left(1-x_{i}(t)-y_{i}(t)\right)-x_{i}(t)(4) \\
& =: F_{i}\left(\mathbf{x}(t), \mathbf{y}(t) ; G^{x}\right) \\
\dot{y}_{i}(t)= & \left(\sum_{j \in V} \gamma^{y}(j, i) y_{j}(t)\right)\left(1-x_{i}(t)-y_{i}(t)\right)-y_{i}(t)(5) \\
& =: F_{i}\left(\mathbf{x}(t), \mathbf{y}(t) ; G^{y}\right),
\end{aligned}
$$

for $i=1, \ldots, N$ with $N=|V|$, with initial condition

$$
\mathbf{z}_{0}=\left(\mathbf{x}_{0}, \mathbf{y}_{0}\right) \in \mathcal{D}_{2}(V)
$$

and underlying bilayer e-network $G^{x, y}=\left(G^{x}, G^{y}\right)$ with $G^{x}=\left(V, \gamma^{x}\right)$ and $G^{y}=\left(V, \gamma^{y}\right)$. For a fixed $G^{x, y}$, we refer to $\left((\mathbf{x}, \mathbf{y})\left(t,\left(\mathbf{x}_{0}, \mathbf{y}_{0}\right) ;\left(G^{x}, G^{y}\right)\right)\right)_{t>0}$ as the flow for $t \geq 0$ of viruses $x$ and $y$ over the corresponding $e$ networks $G^{x}$ and $G^{y}$, starting from $\left(\mathbf{x}_{0}, \mathbf{y}_{0}\right)$ at time $t=$ 0.

The bi-virus dynamics evolves on a bilayer enetwork $G^{x, y}=\left(G^{x}, G^{y}\right)$, where each e-network is associated with the dynamics of a strain of virus as depicted in Fig. 2. Remark that the dynamics of both strains are coupled together, and one cannot in general extract valuable information from the coupled system (4)-(5) by studying the dynamics of each strain separately via (3).

Let $\mathbf{z}_{0}=\left(\mathbf{x}_{0}, \mathbf{y}_{0}\right) \in \mathcal{D}_{2}$ and $G^{x, y}=\left(G^{x}, G^{y}\right) \in$ $\mathcal{E}^{2}$, then, we remark that $x_{i}\left(t, \mathbf{z}_{0}, G^{x, y}\right)$ (respectively, $\left.y_{i}\left(t, \mathbf{z}_{0}, G^{x, y}\right)\right)$ may model the fraction of nodes infected with virus $x$ (respectively, $y$ ) at time $t, t \geq 0$, at island $i$, of a bi-virus epidemics with initial distribution $\mathbf{z}_{0}=\left(\mathbf{x}_{0}, \mathbf{y}_{0}\right)$ and associated complete-multipartite networks whose super-topology plus inter-island rates of infection are given by the e-networks $G^{x}$ and $G^{y}$.

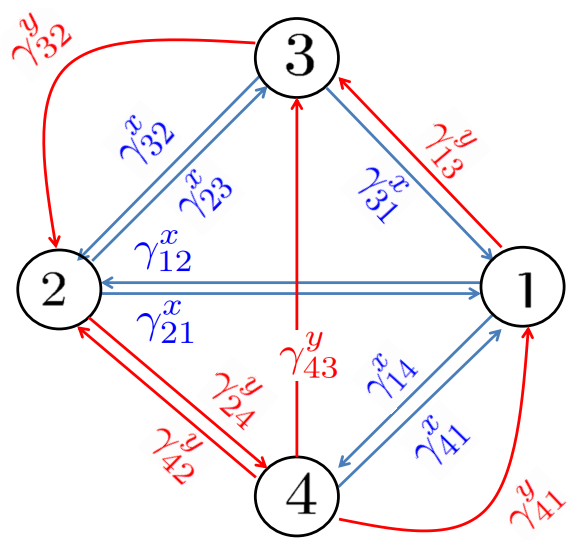

Figure 2: Representation of a bilayer e-network $G^{x, y}=$ $\left(G^{x}, G^{y}\right)$, with $G^{x}=\left(V, \gamma^{x}\right), G^{y}=\left(V, \gamma^{y}\right)$, and $V=$ $\{1,2,3,4\}$. The edges associated with null rates $\gamma$ are not depicted. Each e-network $G^{x}$ and $G^{y}$ is associated with a single strain of virus $x$ and $y$, respectively.

The corresponding flow $x_{i}\left(t, \mathbf{z}_{0}, G^{x, y}\right)$ (respectively, $\left.y_{i}\left(t, \mathbf{z}_{0}, G^{x, y}\right)\right)$ may also model the evolution of the likelihood of infection of node $i$ at time $t$ by virus $x$ (respectively, $y$ ).

For the same reasons as in the single-virus dynamics, the flow $\left((\mathbf{x}, \mathbf{y})\left(t,\left(\mathbf{x}_{0}, \mathbf{y}_{0}\right) ;\left(G^{x}, G^{y}\right)\right)\right)$ is well defined - it is uniquely determined for all time $t, t \geq 0$, as the underlying vector field

$$
\mathbf{F}\left(\cdot, G^{x, y}\right)=\left(\mathbf{F}\left(\cdot, G^{x}\right), \mathbf{F}\left(\cdot, G^{y}\right)\right)
$$

is Lipschitz over the invariant compact domain of interest $\mathcal{D}_{2}$. Again, whenever clear from the context, we will write $y_{i}(t), x_{i}(t)$ or $y_{i}\left(t, \mathbf{z}_{0}\right), x_{i}\left(t, \mathbf{z}_{0}\right)$ instead of $y_{i}\left(t, \mathbf{z}_{0}, G^{x, y}\right)$ or $x_{i}\left(t, \mathbf{z}_{0}, G^{x, y}\right)$. We will also refer to $\stackrel{(n)}{y_{i}}\left(t, \mathbf{z}_{0}, G^{x, y}\right)$ or $\stackrel{(n)}{x_{i}}\left(t, \mathbf{z}_{0}, G^{x, y}\right)$ as the $n$th derivative of the flow to (4)-(5) at time $t, t \geq 0$. Next, we define a class of e-networks, called regular e-networks, where one can fully characterize the qualitative behavior of (4)-(5) namely, determine the attractors and basins of attraction. In words, an e-network is regular if its support graph is regular and the rates between connected nodes $\gamma_{i j}=\gamma$ are constant across edges $(i, j)$.

Definition 7 (Regular E-Network). We call $G:=$ $(V, \gamma) \in \mathcal{E}(V)$ a regular e-network, whenever

$$
\operatorname{supp}(G)=(V, \bar{E})
$$

is a regular graph and

$$
\gamma(i, j)=\gamma \in \mathbb{R}_{++},
$$

for all $(i, j) \in \bar{E}$, i.e., the infection rates are the same across the edges of the underlying support graph. For a regular e-network $G=(V, \gamma)$, we may refer to $\gamma$ as a 
scalar or as the matrix of rates, interchangeably. Further, the regular e-network $G$ is called d-regular, if

$$
d_{i}(G)=|\mathcal{N}(i, G)|=d
$$

for all nodes $i=1, \ldots, N$ and for some $d \in \mathbb{N}$, i.e., all nodes $i \in V$ have degree $d$. We denote by $\mathcal{R}(V) \subset \mathcal{E}(V)$ the subset of regular e-networks with node set $V$.

As we explore later, the qualitative dynamics of (3) or (4)-(5) can be well understood for regular e-networks. Also, the flow in (3) or (4)-(5) over an e-network $G$ or bilayer e-network $G^{x, y}$ can be appropriately lower/upper bounded (in a sense that will be precise later) by flows over sub (inner)/supper (outer) e-networks of $G$ or $G^{x, y}$. We will be able to show fairly general qualitative results for arbitrary e-networks $G$ or $G^{x, y}$ on the infection dynamics by upper/lower bounding its dynamics by the flow over well understood classes of regular sub/supper e-networks that we refer to as inner/outer regular enetworks of $G$ or $G^{x, y}$. Next, we define inequalities that will provide us with the appropriate notion of dominance of a virus (or an e-network) over another virus.

Definition 8 (Inequalities). Let $G^{1}=\left(V, \gamma^{1}\right)$ and $G^{2}=$ $\left(V, \gamma^{2}\right)$ be two e-networks.

- We say that $G^{1} \leq G^{2}$ whenever $\operatorname{supp}\left(G^{1}\right)$ is a subgraph of $\operatorname{supp}\left(G^{2}\right)$.

- We say that $G^{1} \preceq G^{2}$ whenever $\gamma^{1} \leq \gamma^{2}$, where the latter inequality is pointwise.

- We say that $G^{1} \prec G^{2}$ whenever $\gamma^{1}<\gamma^{2}$.

Note that the inequality $\preceq$ is stronger than $\leq$. In words, $G^{1} \preceq G^{2}$ if the rates of infection underlying $G^{1}$ lower-bound the rates of infection of $G^{2}$ at every edge.

Definition 9 ( $\star$-Inequality for Regular E-networks). Let $G^{1}=\left(V, \gamma^{1}\right)$ and $G^{2}=\left(V, \gamma^{2}\right)$ be two regular $e$ networks. We say that $G^{1} \preceq^{\star} G^{2}$, in words, the regular e-network $G^{2} \star$-dominates the regular e-network $G^{1}$, whenever $\gamma^{1} d^{1} \leq \gamma^{2} d^{2}$, where $\gamma^{i} \in \mathbb{R}_{++}$and $d^{i}$ are the rate (constant across edges) and degree associated with the regular e-network $G^{i}$.

Definition 10 (Inner/Outer Regular E-Network). Let $G=(V, \gamma)$ be an e-network. We denote

$$
\underline{G} \in \arg \max \{S: S \preceq G, S \in \mathcal{R}(V)\}
$$

as a maximal regular e-network dominated by G. Correspondingly,

$$
\bar{G} \in \arg \min \{S: S \succeq G, S \in \mathcal{R}(V)\}
$$

is defined as a minimal regular e-network that dominates $G$. We refer to $\underline{G}$ and $\bar{G}$ as an inner and outer, respectively, regular e-networks of $G$. Note that if $G$ is a regular e-network then, $\underline{G}$ and $\bar{G}$ are unique (up to a possible relabeling). In general, $\underline{G}$ and $\bar{G}$ are not uniquely determined.

Since the complete graph is regular and it is a supper graph for any graph, any graph admits a trivial regular outer-graph. Similarly, the trivial e-network of isolated nodes

$$
\underline{G}=\left(V, \mathbf{0}_{N \times N}\right)
$$

i.e., with no connections $(\gamma(i, j)=0$ for all $i, j \in V)$, is an inner-graph of any graph. But the picture is better than it sounds. Reference [22] establishes that a typical large (Erdos-Rényi random) graph admits regular (non trivial) subgraphs with high probability.

Next, we introduce the most important order on the set of e-networks $\mathcal{E}(V)$ in this paper.

Definition 11 ( $\star$-Inequality for General E-networks). Let $G^{1}=\left(V, \gamma^{1}\right)$ and $G^{2}=\left(V, \gamma^{2}\right)$ be two e-networks. We say that

$$
G^{1} \preceq^{\star} G^{2}
$$

whenever

$$
\bar{G}^{1} \varliminf^{\star} \underline{G}^{2}
$$

for some outer and inner regular e-networks $\bar{G}^{1}, G^{2}$ of $G^{1}$ and $G^{2}$, respectively. That is, we say that $G^{2} \star-$ dominates $G^{1}$, whenever there exists a non-trivial innerregular e-network $\underline{G}^{2}$ of $G^{2}$ that $\star$-dominates (in the sense of definition 9) some non-trivial outer-regular $e$ network $\bar{G}^{1}$ of $G^{1}$, i.e., $\bar{\gamma}^{1} \bar{d}^{1} \leq \underline{\gamma}^{2} \underline{d}^{2}$.

Note that definition 11 extends the definition 9 for regular e-networks to general e-networks. One can readily check that ' $\preceq$ ', defines a strict-order (irreflexive and transitive) on the set of non-regular e-networks $\mathcal{E}(V) \backslash \mathcal{R}(V)$, and a preorder (reflexive and transitive) when restricted to the regular e-networks. We shall stress that the inequality ' $\preceq$ ' is not stronger than ' $\preceq$ ', i.e., one may find e-networks $G^{y}$ and $G^{x}$ for which

$$
G^{y} \preceq^{\star} G^{x} \text {, but } G^{y} \npreceq G^{x} .
$$

Fig. 3 illustrates a counter-example, with $G^{y}$ and $G^{x}$ being regular e-networks with degrees $d^{y}=2$ and $d^{x}=$ 3. Thus, $G^{y} \preceq^{\star} G^{x}$, but $G^{y} \npreceq G^{x}$ as, for instance, $\gamma_{12}^{x}=0<\gamma_{12}^{y}$.

\section{Model Analysis - Single-Virus}

In this Section, we present an overview of our approach addressing, for simplicity, the single-virus epidemics. We do not prove the Theorems in this Section as they are trivial Corollaries to the Theorems proved in Section IV for bi-virus over general bilayer e-networks, where our contribution lies. More precisely, Theorem 12 below is a single-virus version of the survival of the fittest Theorem, Theorem 20, in Section IV. 


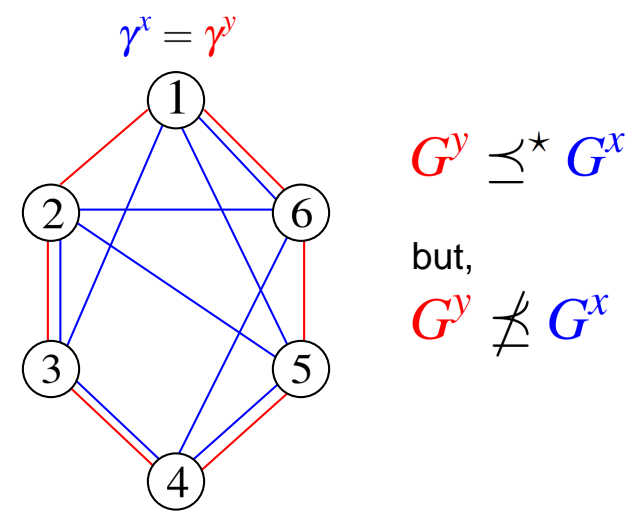

Figure 3: Representation of a bilayer e-network $G^{x, y}=$ $\left(G^{x}, G^{y}\right)$, where the layers $G^{x}$ (in blue/lighter color) and $G^{y}$ (in red/darker color) are regular e-networks with degrees $d^{x}=3$ and $d^{y}=2$, and identical rate parameters. Only edges with positive rate weights are depicted. For instance, $\gamma_{12}^{x}=\gamma_{21}^{x}=0$ as the corresponding edge connecting nodes 1 and 2 are not represented in the support graph of $G^{x}$.

Theorem 12. Let $G$ be an e-network. Let $\underline{G}=(V, \underline{\gamma})$ and $\bar{G}=(V, \bar{\gamma})$ be inner-regular and outer-regular $e$ networks of $G$. If $\underline{\gamma} d(\underline{G})>1$, then

$$
\begin{aligned}
1-\frac{1}{\underline{\gamma} d(\underline{G})} & \leq \liminf _{t \rightarrow \infty} \mathbf{y}\left(t, \mathbf{y}_{0}, G\right) \\
& \leq \limsup _{t \rightarrow \infty} \mathbf{y}\left(t, \mathbf{y}_{0} ; G\right) \leq 1-\frac{1}{\bar{\gamma} d(\bar{G})} .
\end{aligned}
$$

If $\bar{\gamma} d(\bar{G}) \leq 1$, then

$$
\lim _{t \rightarrow \infty} \mathbf{y}\left(t, \mathbf{y}_{0}, G\right) \longrightarrow \mathbf{0} .
$$

Theorem 12 states that if the effective rate $\underline{\gamma} d(\underline{G})>1$ then, the set

$$
\mathcal{A}=\left[1-\frac{1}{\underline{\gamma} d(\underline{G})}, 1-\frac{1}{\bar{\gamma} d(\bar{G})}\right]^{N}
$$

is an attractor to (3) with basin of attraction

$$
\mathcal{B}=\left\{\mathbf{y}_{0} \in \mathcal{D}(V): \mathbf{y}_{0} \neq 0\right\} .
$$

If otherwise $\bar{\gamma} d(\bar{G}) \leq 1$, then the origin is the corresponding global attractor.

Regular E-networks. We do not prove here the Theorem, but sketch its proof, starting with the simpler case of regular e-networks. In this case, clearly, the lower and upper bounds in Theorem 12 coincide.

Let $G=(V, \gamma)$ be regular-i.e., $\operatorname{supp}(G)$ is a regular graph and $\gamma \equiv \gamma_{0}$ is constant across the edges- and assume a symmetric initial condition $\mathbf{y}_{0}=\mathbf{1} y_{0}$, with $y_{0} \in[0,1]$ and $\mathbf{1} \in \mathbb{R}^{N}$ being the $N$-dimensional vector with all entries equal to one. Then, $\left(\mathbf{y}\left(t, y_{0} \mathbf{1} ; G\right)\right)$ is solution to

$$
\dot{z}(t) \mathbf{1}_{N}=\left[d \gamma_{0} z(t)(1-z(t))-z(t)\right] \mathbf{1}_{N}
$$

where $d$ is the degree of the regular e-network $G$. In other words,

$$
y_{1}\left(t, y_{0} \mathbf{1} ; G\right)=\ldots=y_{N}\left(t, y_{0} \mathbf{1} ; G\right) \quad \forall t \geq 0 .
$$

It is easy to study the one-dimensional system (8) and in particular to show that if $\gamma_{0}>1$ and $y_{0} \neq 0$ then,

$$
\mathbf{y}(t) \longrightarrow\left(1-\frac{1}{d \gamma}\right) \mathbf{1},
$$

otherwise,

$$
\mathbf{y}(t) \longrightarrow \mathbf{0} .
$$

The next Theorem allows us to drop the previous assumption on the symmetry of the initial conditions and thus, fully characterize the global attractor of the dynamical system (3), when the underlying e-network is regular.

Theorem 13 (Monotonicity on the initial conditions). Let $G$ be any e-network and $y_{0} \leq y(0)$. Then,

$$
\mathbf{y}\left(t, \mathbf{y}_{0}, G\right) \leq \mathbf{y}(t, \mathbf{y}(0), G), \quad \forall t \geq 0 .
$$

In other words, the Theorem states that the flow $\mathbf{y}\left(t, \mathbf{y}_{0}, G\right)$ preserves the order of initial conditions. Now, assume again that $G$ is a regular e-network. We can combine the analysis in the beginning of this sketchproof with Theorem 13 to yield Theorem 14 .

Theorem 14 (Regular e-network). Let $G=(V, \gamma)$ be a regular e-network. If

$$
\gamma>1 \text { and } \mathbf{y}_{0} \neq \mathbf{0} \in \mathbb{R}^{N}
$$

then,

$$
\mathbf{y}\left(t, \mathbf{y}_{0}, G\right) \longrightarrow\left(1-\frac{1}{d \gamma}\right) \mathbf{1},
$$

otherwise,

$$
\mathbf{y}\left(t, \mathbf{y}_{0}, G\right) \longrightarrow \mathbf{0} .
$$

Indeed, let $\gamma d>1$ and $G$ be regular. If for some $\epsilon>0$ we have $\mathbf{y}_{0}>\epsilon \mathbf{1}$, then,

$$
\mathbf{y}\left(t, \mathbf{y}_{0}, G\right) \geq \mathbf{y}(t, \epsilon \mathbf{1}, G) \longrightarrow\left(1-\frac{1}{d \gamma}\right) \mathbf{1} .
$$

On the other hand,

$$
\mathbf{y}\left(t, \mathbf{y}_{0}, G\right) \leq \mathbf{y}(t, \mathbf{1}, G) \longrightarrow\left(1-\frac{1}{d \gamma}\right) \mathbf{1}
$$

Therefore, Theorem 14 is established for strictly positive initial conditions $\mathbf{y}_{0}>0$. To further prove the Theorem for the more general case where $\mathbf{y}_{0} \neq 0$ (with possibly $y_{0 i}=0$ for some node $i$ ), we need to combine 
the next Theorem, Theorem 15, with Theorem 22 in Section V. Theorem 15 states that a node $i$ sitting $n$ hops away from a node $j$ impacts node $j$ via its $n$th (or higher) order derivatives. In words, $n$th order neighbors of $j$ affect only the $n$th (or higher) order derivative of $\left(y_{j}(t)\right)$.

Theorem 15. Let $y_{i}(t)>0$ and $y_{j}(t)=0, \forall j \neq i$ for some time $t \geq 0$. Then, $\forall \ell<n$

$$
j \in \mathcal{N}^{n}(i) \Rightarrow \stackrel{(n)}{y}(t)>0 \text { and } \stackrel{(\ell)}{y_{j}}(t)=0 .
$$

More precisely, if some entry of the initial condition vector $\mathbf{y}_{0}$ is positive, then $\stackrel{(n)}{\mathbf{y}}\left(t, \mathbf{y}_{0}, G\right)>0$ for some $n \in \mathbb{N}$, as the e-network $G$ is assumed to be connected and finite. Now, Theorem 22 implies that

$$
\mathbf{y}\left(t, \mathbf{y}_{0}, G\right)>0, \forall t \in(0, \delta)
$$

for some $\delta>0$. Therefore, for $t_{0} \in(0, \delta)$, we have

$$
\begin{aligned}
\liminf _{t \rightarrow \infty} \mathbf{y}\left(t, \mathbf{y}_{0}\right) & =\liminf _{t \rightarrow \infty} \mathbf{y}\left(t, \mathbf{y}\left(t_{0}, \mathbf{y}_{0}\right)\right) \\
& \geq \lim _{t \rightarrow \infty} \mathbf{y}(t, \epsilon \mathbf{1}) \longrightarrow\left(1-\frac{1}{d \gamma}\right) \mathbf{1},
\end{aligned}
$$

for some $\epsilon>0$ (that depends on the choice of $t_{0}$ ). Thus, Theorem 14 is proved and we have a complete qualitative characterization of the ODE (3) when the underlying enetwork $G$ is regular.

Monotonicity under the partial order ' $\preceq$ ' on the set of e-networks. Now, we observe that the flow $\left(\mathbf{y}\left(t, \mathbf{y}_{0}, G\right)\right)$ to (3) also preserves upper/lower bounds - with respect to the partial order ' $\preceq$ ' - on enetworks.

Theorem 16. Let $G_{1}=\left(V, \gamma^{(1)}\right) \preceq G_{2}=\left(V, \gamma^{(2)}\right)$ be two e-networks and $y_{0} \leq y(0)$. Then,

$$
\mathbf{y}\left(t, \mathbf{y}_{0}, G_{1}\right) \leq \mathbf{y}\left(t, \mathbf{y}(0), G_{2}\right), \quad \forall t \geq 0 .
$$

This Theorem combined with Theorem 14 readily implies Theorem 12. Indeed, let

$$
\underline{G} \preceq G \preceq \bar{G}
$$

where

$$
\underline{G}=(V, \underline{\gamma}) \text { and } \bar{G}=(V, \bar{\gamma})
$$

are inner and outer, respectively, regular e-networks for $G$. Assume, for instance, that $\underline{\gamma} d(\underline{G})>1$. Then,

$$
\begin{aligned}
1-\frac{1}{\underline{\gamma d}(\underline{G})} & =\lim _{t \rightarrow \infty} \mathbf{y}\left(t, \mathbf{y}_{0}, \underline{G}\right) \\
& \leq \liminf _{t \rightarrow \infty} \mathbf{y}\left(t, \mathbf{y}_{0}, G\right) \\
& \leq \limsup _{t \rightarrow \infty} \mathbf{y}\left(t, \mathbf{y}_{0}, G\right) \\
& \leq \lim _{t \rightarrow \infty} \mathbf{y}\left(t, \mathbf{y}_{0}, \bar{G}\right)=1-\frac{1}{\bar{\gamma} d(\bar{G})},
\end{aligned}
$$

where the first and last equalities hold from Theorem 14, and the first and third inequalities hold from Theorem 16.

In the next Section, we formally prove survival of the fittest (Theorem 20) through exploring a generalization of the properties studied in this Section: (i) we establish survival of the fittest on regular e-networks; (ii) we prove that the system is monotonous in a certain sense; (iii) we combine (i) and (ii) jointly with a new order ' $\preceq$. The Theorems in Section IV generalize the Theorems discussed in this Section, and they will be formally proved.

\section{General E-Networks: Bi-Virus}

The qualitative analysis of dynamical systems comprises characterizing their attractors and corresponding basins of attraction as depicted in the previous Section. In general, this is achieved by either Lyapunov theory or numerical simulations. For instance, reference [12] studies the qualitative behavior of the single virus dynamics (3) via a Lyapunov stability approach. In contrast, in this paper, we are interested in studying the bi-virus dynamics (4)-(5). Our qualitative analysis of (4)-(5) does not follow from the single-virus results in [12]. Indeed, the results in [12] rely on the irreducibility of the matrix $\gamma=\left(\gamma_{i j}\right)_{i j}$ underlying the linear term of the vector field

$$
\mathbf{F}(\mathbf{y})=\left(\gamma-I_{N \times N}\right) \mathbf{y}-N(\mathbf{y})
$$

for the single virus ODE (3), where

$$
N(\mathbf{y})=(\gamma \mathbf{y}) \odot \mathbf{y}
$$

comprises the nonlinear term of $\mathbf{F}(\mathbf{y})$ in (3), and $\odot$ is the Hadamard pointwise product. If $\gamma$ is reducible then, one can still study the single-virus dynamics at each of the connected components individually (the system is uncoupled) as the nonlinear term $N(\mathbf{y})$ decouples accordingly. For the bi-virus case, the linear term of

$$
\mathbf{F}(\mathbf{z})=\left(\gamma^{x, y}-I_{2 N \times 2 N}\right) \mathbf{z}-\widetilde{N}(\mathbf{z})
$$

is reducible, with $\mathbf{z}:=(\mathbf{x}, \mathbf{y})$, but the nonlinear term $\widetilde{N}$ still couples the epidemics dynamics in each network connected component associated to $\gamma^{x}$ and $\gamma^{y}$. Therefore, its dynamics cannot be studied independently for each subsystem and the Lyapunov methods of [12] that relied on the matrix $\gamma^{x, y}$ being irreducible do not apply. So, we need to develop new methodologies to analyze the global stability and qualitative behavior of (4)-(5).

To prove the survival of the fittest, Theorem 20, we follow similar steps as in the previous Section: study the ODE (4)-(5) on regular e-networks, and then we prove the result on general e-networks by combining the analysis in regular e-networks with a skewed-monotonous property of the dynamical system (4)-(5), as will be made 
clearer. Also, we resort to the inequality ' $\preceq$ ' in the set of e-networks introduced in definition 11, in Section II.

The next Lemma represents a simpler version of the main Theorem, Theorem 20, when symmetry is assumed. Namely, it observes that if all nodes are evenly infected with the virus strains $x$ and $y$ in regular e-networks $G^{x}$ and $G^{y}$ then, they will remain equally infected for all time $t$, and moreover the weaker strain dies out. Remark that

$$
\begin{gathered}
\left(\mathbf{x}\left(t,\left(\mathbf{x}_{0}, \mathbf{y}_{0}\right) ; G^{x, y}\right)\right) \\
\text { and } \\
\left(\mathbf{y}\left(t,\left(\mathbf{y}_{0}, \mathbf{y}_{0}\right) ; G^{x, y}\right)\right)
\end{gathered}
$$

stand for the flows to the ODE (4)-(5) associated with the strains $x$ and $y$, respectively, over the bilayer e-network $G^{x, y}$, for $t \geq 0$, and starting from the state $\left(\mathbf{x}_{0}, \mathbf{y}_{0}\right)$ at time $t=0$.

Lemma 17. Let $G^{x}, G^{y} \in \mathcal{R}(V)$ be two regular enetworks. Let $x_{0}, y_{0} \in \mathbb{R}$. Then,

$\mathbf{x}\left(t,\left(x_{0}, y_{0}\right) \otimes \mathbf{1}_{N} ; G^{x, y}\right)=x\left(t,\left(x_{0}, y_{0}\right) ; G^{x, y}\right) \mathbf{1}_{N}$ $\mathbf{y}\left(t,\left(x_{0}, y_{0}\right) \otimes \mathbf{1}_{N} ; G^{x, y}\right)=y\left(t,\left(x_{0}, y_{0}\right) ; G^{x, y}\right) \mathbf{1}_{N}$

for all $t, t \geq 0$, where ' $\otimes$ ' is the Kronecker product. In this case, if $\gamma_{0}^{x} d^{x}>\gamma_{0}^{y} d^{y}$ with $\gamma_{0}^{x}>\frac{1}{d^{x}}$, we have

$$
\begin{aligned}
& \mathbf{x}(t) \longrightarrow\left(1-\frac{1}{\gamma_{0}^{x} d\left(G^{x}\right)}\right) \mathbf{1}_{N} \\
& \mathbf{y}(t) \longrightarrow \mathbf{0}_{N}
\end{aligned}
$$

otherwise, if $\gamma_{0}^{x} \leq \frac{1}{d}$, then

$$
\begin{aligned}
& \mathbf{x}(t) \longrightarrow \mathbf{0}_{N} \\
& \mathbf{y}(t) \longrightarrow \mathbf{0}_{N} .
\end{aligned}
$$

Proof. When the nodes are evenly infected, i.e.,

$$
\mathbf{y}(0)=y_{0} \mathbf{1} \text { and } \mathbf{x}(0)=x_{0} \mathbf{1},
$$

and provided that the underlying e-networks $G^{x}$ and $G^{y}$ are regular then, the flow

$$
\left((\mathbf{x}, \mathbf{y})\left(t,\left(x_{0}, y_{0}\right) \otimes \mathbf{1} ; G^{x, y}\right)\right)
$$

reduces to the solution of the $2 \mathrm{D}$ ODE

$$
\begin{aligned}
& \dot{y}(t) \mathbf{1}=\left(\gamma_{0}^{y} d\left(G^{y}\right) y(t)(1-x(t)-y(t))-y(t)\right) \mathbf{1} \\
& \dot{x}(t) \mathbf{1}=\left(\gamma_{0}^{x} d\left(G^{x}\right) x(t)(1-x(t)-y(t))-x(t)\right) \mathbf{1} .
\end{aligned}
$$

The ODE (13)-(14) also describes the dynamics of diffusion of two strains of virus in a self-linked singlenode e-network and it was studied in Reference [23], from where the asymptotics follows.

The next Theorem is an extension of the monotonous property for a single virus in Theorem 16 to the bi-virus epidemics case: skewed bounds on the initial conditions and e-networks are preserved by the flow of the dynamical system (4)-(5).

Theorem 18 (Bi-virus Monotonicity). Let $G^{x, y}=$ $\left(G^{x}, G^{y}\right)$ and $G_{s, S}^{x, y}=\left(G_{s}^{x}, G_{S}^{y}\right)$ be two bilayer enetworks with

$$
G_{s}^{x} \preceq G^{x} \text { and } G_{S}^{y} \succeq G^{y} .
$$

Define the initial conditions

$$
\mathbf{z}_{0}=\left(\mathbf{x}_{0}, \mathbf{y}_{0}\right) \text { and } \mathbf{z}(0)=(\mathbf{x}(0), \mathbf{y}(0)),
$$

with $\mathbf{x}_{0} \leq \mathbf{x}(0)$ and $\mathbf{y}_{0} \geq \mathbf{y}(0)$. Then,

$$
\begin{aligned}
& \mathbf{y}\left(t, \mathbf{z}_{0}, G_{s, S}^{x, y}\right) \geq \mathbf{y}\left(t, \mathbf{z}(0), G^{x, y}\right) \\
& \mathbf{x}\left(t, \mathbf{z}_{0}, G_{s, S}^{x, y}\right) \leq \mathbf{x}\left(t, \mathbf{z}(0), G^{x, y}\right)
\end{aligned}
$$

for all time $t, t \geq 0$.

Proof. Assume that

$$
\mathbf{y}(0) \neq \mathbf{y}_{0} \text { or } \mathbf{x}(0) \neq \mathbf{x}_{0} .
$$

We write $\mathbf{y}\left(t, \mathbf{z}_{0}\right), \mathbf{x}\left(t, \mathbf{z}_{0},\right)$ instead of $\mathbf{y}\left(t, \mathbf{z}_{0}, G_{s, S}^{x, y}\right)$ and $\mathbf{x}\left(t, \mathbf{z}_{0}, G_{s, S}^{x, y}\right)$; or $\mathbf{y}(t, \mathbf{z}(0)), \mathbf{x}(t, \mathbf{z}(0))$ instead of $\mathbf{y}\left(t, \mathbf{z}(0), G^{x, y}\right)$ and $\mathbf{x}\left(t, \mathbf{z}(0), G^{x, y}\right)$. Define

$$
\begin{aligned}
& T=\inf \left\{t: t \geq 0, \mathbf{y}(t, \mathbf{z}(0)) \not \leq \mathbf{y}\left(t, \mathbf{z}_{0}\right)\right. \text { or } \\
& \left.\mathbf{x}(t, \mathbf{z}(0)) \nsupseteq \mathbf{x}\left(t, \mathbf{z}_{0}\right)\right\} .
\end{aligned}
$$

Assume that $T<\infty$. Then, for

$$
i, j \in\{1, \ldots, M\} \text { with } i \neq j,
$$

we have one of the following:

$$
\begin{aligned}
& y_{i}(T, \mathbf{z}(0))=y_{i}\left(T, \mathbf{z}_{0}\right) \text { and } y_{j}(T, \mathbf{z}(0))<y_{j}\left(T, \mathbf{z}_{0}\right) \\
& x_{i}(T, \mathbf{z}(0))=x_{i}\left(T, \mathbf{z}_{0}\right) \text { and } x_{j}(T, \mathbf{z}(0))>x_{j}\left(T, \mathbf{z}_{0}\right) .
\end{aligned}
$$

Without loss of generality, choose configuration (17) and assume $j \in \mathcal{N}^{n}(i)$ is the closest node to $i$ where we have strict inequality $y_{j}(T, \mathbf{z}(0))<y_{j}\left(T, \mathbf{z}_{0}\right)$.

Case 1: If $x_{1}(T, \mathbf{z}(0))+y_{1}(T, \mathbf{z}(0))<1$, then, from Theorem 24 we have

$$
\stackrel{(n)}{y_{i}}(T, \mathbf{z}(0))<\stackrel{(n)}{\widetilde{y}_{i}}\left(T, \mathbf{z}_{0}\right) .
$$

Therefore, from Theorem 22 in Section V, we have that

$\exists \epsilon_{1}>0: y_{1}(t, \mathbf{z}(0))<y_{1}\left(t, \mathbf{z}_{0}\right), \quad \forall T<t<T+\epsilon_{1}$.

Also,

$$
\begin{gathered}
y_{j}(T, \mathbf{z}(0))<y_{j}\left(T, \mathbf{z}_{0}\right) \Rightarrow \\
\exists \epsilon_{2}>0: y_{j}(t, \mathbf{z}(0))<y_{j}\left(t, \mathbf{z}_{0}\right), \forall T<t<T+\epsilon_{2} .
\end{gathered}
$$

Thus,

$$
\mathbf{y}(t, \mathbf{z}(0)) \leq \mathbf{y}\left(t, \mathbf{z}_{0}\right), \quad \forall \quad T<t<T+\epsilon
$$


with $\epsilon=\epsilon_{1} \wedge \epsilon_{2}$. Similarly, we have that

$$
\mathbf{x}(t, \mathbf{z}(0)) \geq \mathbf{x}\left(t, \mathbf{z}_{0}\right), \quad \forall T<t<T+\alpha
$$

for some $\alpha>0$.

Case 2: If $x_{1}(T, \mathbf{z}(0))+y_{1}(T, \mathbf{z}(0))=1$, then,

$$
\begin{gathered}
\dot{x}_{1}(T, \mathbf{z}(0))+\dot{y}_{1}(T, \mathbf{z}(0))=-\left(x_{1}(T)+y_{1}(T)\right)<0 \\
\Downarrow \\
\exists \epsilon>0: x_{1}(t, \mathbf{z}(0))+y_{1}(t, \mathbf{z}(0))<1
\end{gathered}
$$

for all $t \in(T, T+\epsilon)$. In any case, we reach a contradiction on the definition of $T$, and the Theorem is proved.

We can now drop the symmetry on the initial conditions assumed in Lemma 17.

Theorem 19 (Regular bilayer e-network). Let $G^{x, y}=$ $\left(G^{x}, G^{y}\right) \in \mathcal{E}^{2}(V)$ be regular e-networks with $\gamma^{x} \equiv \gamma_{0}^{x}$ and $\gamma^{y} \equiv \gamma_{0}^{y}$. Let $\mathbf{z}_{0}=\left(\mathbf{x}_{0}, \mathbf{y}_{0}\right)$ be the initial condition with $\mathbf{x}_{0} \neq 0$. If $G^{y} \preceq^{\star} G^{x}$ and $\gamma_{0}^{x} d^{x}>1$ then,

$$
\begin{aligned}
& \mathbf{x}\left(t, \mathbf{z}_{0} ; G^{x, y}\right) \longrightarrow\left(1-\frac{1}{d^{x} \gamma_{0}^{x}}\right) \mathbf{1}_{N} \\
& \mathbf{y}\left(t, \mathbf{z}_{0} ; G^{x, y}\right) \longrightarrow \mathbf{0}_{N},
\end{aligned}
$$

otherwise, if $\gamma_{0}^{x} d^{x} \leq 1$

$$
\mathbf{x}\left(t, \mathbf{z}_{0} ; G^{x, y}\right) \longrightarrow \mathbf{0}_{N} .
$$

In words, if in a bilayer regular e-network the strain $y$ is $\star$-weaker than the strain $x$, then the strain $y$ dies out, whereas the $\star$-stronger strain persists, if strong enough.

Proof. First, let us assume that $\mathbf{x}_{0}>\epsilon \mathbf{1}>0$ for some positive $\epsilon$ (all entries are strictly positive). Define the symmetric initial conditions

$$
\begin{aligned}
& \mathbf{z}_{0}^{1}=\left(\mathbf{x}_{0}^{1}, \mathbf{y}_{0}^{1}\right):=(\epsilon \mathbf{1}, \mathbf{1}) \in \mathbb{R}^{N} \times \mathbb{R}^{N} \\
& \mathbf{z}_{0}^{2}=\left(\mathbf{x}_{0}^{2}, \mathbf{y}_{0}^{2}\right):=(\mathbf{1}, \mathbf{0}) \in \mathbb{R}^{N} \times \mathbb{R}^{N} .
\end{aligned}
$$

If $\gamma^{x} d^{x}>1$ then,

$$
\begin{aligned}
1-\frac{1}{\gamma^{x} d^{x}} & =\lim _{t \rightarrow \infty} \mathbf{x}\left(t, \mathbf{z}_{0}^{1}, G^{x, y}\right) \\
& \leq \liminf _{t \rightarrow \infty} \mathbf{x}\left(t, \mathbf{z}_{0}, G^{x, y}\right) \\
& \leq \limsup _{t \rightarrow \infty} \mathbf{x}\left(t, \mathbf{z}_{0}, G^{x, y}\right) \\
& \leq \lim _{t \rightarrow \infty} \mathbf{x}\left(t, \mathbf{z}_{0}^{2}, G^{x, y}\right)=1-\frac{1}{\gamma^{x} d^{x}}
\end{aligned}
$$

and

$$
\begin{aligned}
\limsup _{t \rightarrow \infty} \mathbf{y}\left(t, \mathbf{z}_{0}, G^{x, y}\right) & \leq \lim _{t \rightarrow \infty} \mathbf{y}\left(t, \mathbf{z}_{0}^{1}, G^{x, y}\right)(24 \\
& =\mathbf{0}
\end{aligned}
$$

where the first and last equalities in equations (20)-(23) hold from Lemma 17, and the first and third inequalities in equations (20)-(23) hold from Theorem 18. Equations (24)-(25) are now obvious.

Now, assume $\mathbf{x}_{0} \neq 0$, with some entry possibly equal to zero. Theorems 22 and 24 (refer to Section V) imply that there exists $\delta>0$ such that

$$
\mathbf{x}\left(t, \mathbf{z}_{0}, G^{x, y}\right)>0 \forall t \in(0, \delta) .
$$

Choose $t_{0} \in(0, \delta)$, and we have

$$
\begin{aligned}
\lim _{t \rightarrow \infty} \mathbf{x}\left(t, \mathbf{z}_{0}\right) & =\lim _{t \rightarrow \infty} \mathbf{x}\left(t, \mathbf{z}\left(t_{0}, \mathbf{z}_{0}\right)\right) \\
& =\left(1-\frac{1}{d \gamma}\right) \mathbf{1},
\end{aligned}
$$

where the first equality is due to the semi-group property associated with the flow to autonomous ODEs, and the second equality holds from our previous argument that assumed $\mathbf{x}_{0}>0$. In other words, as long as there is some - possibly tiny - degree of infection of the stronger virus strain $x$ in the bilayer regular e-network, such strain will prevail eventually, with a degree of infection given by the equation (28).

As a simple illustration, Fig. 4 shows the possibility of bounding any configuration by simpler symmetric wellcharacterized configurations. Such bounds are preserved for all $t, t \geq 0$ as established in Theorem 18 .

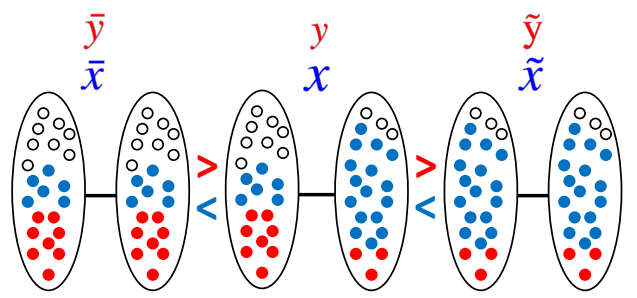

Figure 4: Population of blue (lighter color) in the center bipartite network is lower and upper bounded by the corresponding populations in the left and right bipartite networks. The same goes, in the other way around, for the red (darker) population. The symmetric configurations in the left and right bipartite networks induce wellknown solutions that bound the qualitative behavior of the middle configuration.

We can now establish the main Theorem of this paper, Theorem 20 .

Theorem 20 (Natural Selection). Let $G^{y} \preceq^{\star} G^{x}$. Then,

$$
\mathbf{y}\left(t, \mathbf{z}_{0} ; G^{x, y}\right) \rightarrow 0
$$


Additionally, if $\underline{\gamma}^{x} \underline{d}^{x}>1$ then,

$$
\begin{aligned}
1-\frac{1}{\underline{\gamma}^{x} \underline{d}^{x}} & \leq \liminf _{t \rightarrow \infty} \mathbf{x}\left(t, \mathbf{z}_{0} ; G^{x, y}\right) \\
& \leq \limsup _{t \rightarrow \infty} \mathbf{x}\left(t, \mathbf{z}_{0} ; G^{x, y}\right) \\
& \leq 1-\frac{1}{\bar{\gamma}^{x} \bar{d}^{x}} .
\end{aligned}
$$

In words, the $\star$-weaker strain $y$ dies out and the $\star$ stronger strain persists if strong enough. For instance, assuming that the ODE (4)-(5) models a Dengue versus vaccines epidemics, then a policy of vaccination that could guarantee the $\star$-inequality

$$
G^{\text {Dengue }} \preceq^{\star} G^{\text {Policy of Vaccination }}
$$

could, in theory, exterminate the epidemics.

Proof. Let $\bar{G}^{y}$ (respectively, $\underline{G}^{x}$ ) be an outer (respectively, inner) regular e-network of $G^{y}$ (respectively, $G^{x}$ ) such that

$$
\bar{G}^{y} \preceq \underline{G}^{x},
$$

which is possible to choose as by assumption $G^{y} \preceq^{\star} G^{x}$. Let also $\bar{G}^{x}$ be an outer regular e-network of $G^{x}$. Define the bilayer e-networks $\underline{G}_{1}^{x, y}:=\left(\underline{G}^{x}, \bar{G}^{y}\right)$ and $\underline{G}_{2}^{x, y}:=$ $\left(\bar{G}^{x}, \mathbf{0}\right)$. If $\underline{\underline{\gamma}}^{x} \underline{d}^{x}>1$, then
\[ \begin{aligned}\left(1-\frac{1}{\underline{\gamma}^{x} \underline{d}^{x}}\right) & =\lim _{t \rightarrow \infty} \mathbf{x}\left(t, \mathbf{z}_{0} ; \bar{G}_{1}^{x, y}\right) \\ & \leq \liminf _{t \rightarrow \infty} \mathbf{x}\left(t, \mathbf{z}_{0} ; G^{x, y}\right) \\ & \leq \limsup _{t \rightarrow \infty} \mathbf{x}\left(t, \mathbf{z}_{0} ; G^{x, y}\right) \\ & \leq \lim _{t \rightarrow \infty} \mathbf{x}\left(t, \mathbf{z}_{0} ; \bar{G}_{2}^{x, y}\right) \\ & =\left(1-\frac{1}{\bar{\gamma}^{x} \bar{d}^{x}}\right)\end{aligned} \]

$$
\limsup _{t \rightarrow \infty} \mathbf{y}\left(t, \mathbf{z}_{0} ; G^{x, y}\right) \leq \lim _{t \rightarrow \infty} \mathbf{y}\left(t, \mathbf{z}_{0} ; \bar{G}_{1}^{x, y}\right)=0,
$$

where the convergences are due to Theorem 19 and the inequalities follow from Theorem 18. The remaining case $\bar{\gamma}^{x} \bar{d}^{x}<1$ is now obvious.

The next Theorem extends Theorem 20 to the case of multivirus epidemics. It states that if $G^{1} \star$-dominates all the other strains in the network then, the weaker strains necessarily die out. The ODE governing the multivirus epidemics is given by,

$$
\frac{d}{d t} y_{i k}(t)=\left(\sum_{j} \gamma_{j i}^{k} y_{j k}(t)\right)\left(1-\sum_{\ell=1}^{K} y_{i \ell}(t)\right)-y_{i k}(t),
$$

for $i=1, \ldots, N$ and $k=1, \ldots, M$, where $y_{i k}(t)$ stands for the degree of infection at node $i$ by virus type $k$. This is the corresponding dynamics obtained from the peerto-peer rules of infection in the limit of large networks for the multivirus case (refer to [7]). Also, for simplicity, we write (with only one sub-index)

$$
\mathbf{y}_{k}(t)=\left(\mathbf{y}_{1 k}(t), \ldots, \mathbf{y}_{N k}(t)\right)
$$

as the vector stacking the degree of infection across nodes due to the virus $k$.

Theorem 21. Let $G^{1} \succeq^{\star} G^{2} \succeq \ldots \succeq G^{M}$ be the e-networks associated with viruses $k=1, \ldots, M$ governed by the ODE (33). Then,

$$
\mathbf{y}_{m}(t) \longrightarrow \mathbf{0}
$$

for all $m \geq 2$. Additionally, if $\underline{\gamma}^{1} \underline{d}^{1}>1$ then, $\left(\mathbf{y}_{1}(t)\right)$ obeys inequalities (29)-(31).

Proof. First, it is easy to check that if $(\mathbf{y}(t))$ is solution to the ODE (33) and if $\mathbf{y}_{k}(0)=\mathbf{0}_{N}$ for some $k \in$ $\{1, \ldots, K\}$ then, $\mathbf{y}_{k}(t)=\mathbf{0}$ for all time $t \geq 0$. In words, if a virus strain is not present in the network at time $t_{0} \geq 0$ then, it will remain extinct for all future times $t \geq t_{0}$. Now, let

$$
\left\{\begin{array}{ll}
\mathbf{y}_{1}(0) & \geq \widetilde{\mathbf{y}}_{1}(0) \\
\sum_{k \neq 1} \mathbf{y}_{k}(0) & \leq \widetilde{\mathbf{y}}_{2}(0)
\end{array} .\right.
$$

Define $(\widetilde{\mathbf{y}}(t)):=\left(\left(\mathbf{y}_{1}, \mathbf{y}_{2}\right)\left(t,\left(\widetilde{\mathbf{y}}_{1}(0), \widetilde{\mathbf{y}}_{2}(0)\right) ; \bar{G}^{1,2}\right)\right)$ as the bi-virus flow over the bilayer regular e-network

$$
\underline{\bar{G}}^{1,2}=\left(\underline{G}_{1}, \bar{G}^{2}\right)
$$

with initial condition $\left(\widetilde{\mathbf{y}}_{1}(0), \widetilde{\mathbf{y}}_{2}(0)\right)$. The inequalities (34) are preserved by the dynamics, i.e.,

$$
\begin{cases}\mathbf{y}_{1}(t) & \geq \widetilde{\mathbf{y}}_{1}(t) \\ \sum_{k \neq 1} \mathbf{y}_{k}(t) & \leq \widetilde{\mathbf{y}}_{2}(t)\end{cases}
$$

for all $t \geq 0$, where $(\mathbf{y}(t))$ and $(\widetilde{\mathbf{y}}(t))$ are solutions to (33) with initial conditions

$$
\mathbf{y}(0) \in \mathbb{R}^{M \times N} \text { and } \widetilde{\mathbf{y}}(0) \in \mathbb{R}^{2 \times N}
$$

obeying inequalities (34). We can establish this fact through similar invariance type of arguments as, for instance, in the proof of Theorem 18: let $T$ be the hitting time to invalidate any of the inequalities in equation (36), assume that $T<\infty$ and reach a contradiction (we do not repeat the steps here). Now, from Theorem 19

$$
\begin{cases}\mathbf{y}_{1}(t) & \geq \tilde{\mathbf{y}}_{1}(t) \rightarrow\left(1-\frac{1}{\underline{\gamma}^{1} d\left(\underline{G}^{1}\right)}\right) \mathbf{1}_{N} \\ \sum_{k \neq 1} \mathbf{y}_{k}(t) & \leq \widetilde{\mathbf{y}}_{2}(t) \rightarrow \mathbf{0}_{N}\end{cases}
$$

The remaining upper/lower bounds can be obtained similarly and the Theorem is proved.

Theorem 20 (or more generally 21) states that 
if $\underline{\gamma}^{x} \underline{d}^{x}>1$ and $G^{y} \preceq^{\star} G^{x}$ then, the set

$$
\mathcal{A}=\left[1-\frac{1}{\underline{\gamma}^{x} d\left(\underline{G}^{x}\right)}, 1-\frac{1}{\bar{\gamma}^{x} d\left(\bar{G}^{x}\right)}\right]^{N} \times\{0\}^{N}
$$

is an attractor to (4)- (5) with basin of attraction $\mathcal{B}=$ $\left\{\left(\mathbf{x}_{0}, \mathbf{y}_{0}\right) \in \mathcal{D}_{2}(V): \mathbf{x}_{0} \neq 0\right\}$. Theorem 19 states that, when symmetry is assumed, namely, $G^{x}$ is regular then, the attractor $\mathcal{A}$ reduces to the singleton

$$
\left\{1-\frac{1}{d\left(G^{x}\right) \gamma^{x}}\right\}^{N} \times\{0\}^{N}
$$

\section{Auxiliary Results}

This Section contains three auxiliary Theorems that are necessary to prove the Theorems in Section IV. We simply enunciate and prove them.

Theorem 22. Let $f:(0,+\infty) \rightarrow \mathbb{R}$ be an analytic function. If for some $T \in \mathbb{R}$ we have

$$
\stackrel{(k)}{f(T)}>0 \text { and } \stackrel{(m)}{f(T)}=0,
$$

$\forall m=0,1, \ldots, k-1$ and $k \geq 1$ then, there exists $\epsilon>0$ such that $f(t)>0$ for all $t \in(T, T+\epsilon)$.

Proof. Without loss of generality, assume $T=0$. Since $f \in C^{\omega}(\mathbb{R})$ then,

$$
\begin{aligned}
f(t) & =f(0)+\dot{f}(0) t+\ddot{f}(0) t^{2}+\ldots+\stackrel{(k)}{f}(0) t^{k}+r(t) \\
& =f(0)+\left(\stackrel{(k)}{f}(0)+\frac{r(t)}{t^{k}}\right) t^{k}
\end{aligned}
$$

with $\frac{|r(t)|}{t^{k}} \rightarrow 0$ as $t \rightarrow 0$. Choose $\delta$ such that

$$
\frac{|r(t)|}{t^{k}}<\frac{(k)}{2}, \forall t \in(0, \delta) .
$$

Then,

$$
\stackrel{(k)}{f}(0)+\frac{r(t)}{t^{k}}>0, \forall t \in(0, \delta) .
$$

Then,

$$
f(t)=f(0)+\left(\stackrel{(k)}{f}(0)+\frac{r(t)}{t^{k}}\right) t^{k}>0, \forall t \in(0, \delta) .
$$

For notational simplicity, in the Theorems 23 and 24 we define the flows

$$
(\mathbf{z}(t))=(\mathbf{x}(t), \mathbf{y}(t)):=\left((\mathbf{x}, \mathbf{y})\left(t, \mathbf{z}(0) ; G^{x, y}\right)\right)
$$

and

$$
(\widetilde{\mathbf{z}}(t))=(\widetilde{\mathbf{x}}(t), \widetilde{\mathbf{y}}(t)):=\left((\widetilde{\mathbf{x}}, \widetilde{\mathbf{y}})\left(t, \widetilde{\mathbf{z}}(0) ; G^{x, y}\right)\right)
$$

over the same bilayer e-network $G^{x, y}$ but, with possibly different initial conditions

$$
\mathbf{z}(0)=(\mathbf{x}(0), \mathbf{y}(0))
$$

and

$$
\widetilde{\mathbf{z}}(0)=(\widetilde{\mathbf{x}}(0), \widetilde{\mathbf{y}}(0))
$$

Theorem 24 extends Theorem 15 for the bi-virus dynamics case. Theorem 24 relies on the next Theorem 23 .

Theorem 23. Let $y_{i}(0)=\widetilde{y}_{i}(0)$ and $x_{i}(0)=\widetilde{x}_{i}(0)$. Let $\overline{\mathcal{N}}^{n}(i):=\bigcup_{\ell=1}^{n} \mathcal{N}^{\ell}(i)$. Then:

$$
\left\{\begin{array}{l}
y_{k}(0)=\widetilde{y}_{k}(0) \\
x_{k}(0)=\widetilde{x}_{k}(0)
\end{array} \quad \forall k \in \overline{\mathcal{N}}^{n}(i)\right.
$$

\section{$\Downarrow$}

$$
\stackrel{(\ell)}{y_{i}}(0)=\stackrel{(\ell)}{\widetilde{y}_{i}}(0) \quad \text { and } \stackrel{(\ell)}{x}_{i}(0)=\stackrel{(\ell)}{\widetilde{x}_{i}}(0), \quad \forall \ell \leq n
$$

Proof. We apply induction on $n$. For $n=1$,

$$
\begin{aligned}
& \frac{d}{d t} y_{i}(t)=\left(\sum_{j} \gamma_{j i}^{y} y_{j}(t)\right)\left(1-x_{i}(t)-y_{i}(t)\right)-y_{i}(t) \\
& \frac{d}{d t} \widetilde{y}_{i}(t)=\left(\sum_{j} \gamma_{j i}^{y} \widetilde{y}_{j}(t)\right)\left(1-\widetilde{x}_{i}(t)-\widetilde{y}_{i}(t)\right)-\widetilde{y}_{i}(t) .
\end{aligned}
$$

Note that $\widetilde{y}_{j}(0)=y_{j}(0), \forall j \in \mathcal{N}(i)$ and $x_{i}(0)=$ $\widetilde{x}_{i}(0)$. By inspection, $\dot{\widetilde{y}}_{i}(0)=\dot{y}_{i}(0)$ and (by assumption) $y_{i}(0)=\widetilde{y}_{i}(0)$.

Now, assume Theorem 23 holds for $n-1$. We establish that it holds for $n$. We have:

$$
\begin{aligned}
& \stackrel{(n)}{y}_{i}(0)=\underbrace{\left(\sum_{j} \gamma_{j i}^{y}{ }^{(n-1)} y_{j}(0)\right)\left(1-y_{i}(0)-x_{i}(0)\right)}_{\mathbf{A}} \\
& -\underbrace{\sum_{\ell=1}^{n-1}\left(\begin{array}{c}
n-1 \\
\ell
\end{array}\right) \stackrel{(\ell)}{y}_{i}(0)\left(\sum_{q} \gamma_{q i}^{y}{ }^{(n-1-\ell)} y_{q}^{(0)}\right)}_{\mathbf{B}} \\
& -\underbrace{\left(\begin{array}{c}
(n-1) \\
y_{i}(0)
\end{array}\right.}_{\mathbf{C}} \\
& -\underbrace{\sum_{\ell=1}^{n-1}\left(\begin{array}{c}
n-1 \\
\ell
\end{array}\right) \stackrel{(\ell)}{x_{i}(0)}\left(\sum_{j} \gamma_{j i}^{y} \begin{array}{c}
(n-1-\ell) \\
y_{j}(0)
\end{array}\right)}_{\mathbf{D}} \\
& \stackrel{(n)}{y}_{i}(0)=\underbrace{\left(\sum_{j} \gamma_{j i}^{y} \stackrel{(n-1)}{\left.\widetilde{y}_{j}(0)\right)}\left(1-\widetilde{y}_{i}(0)-\widetilde{x}_{i}(0)\right)\right.}_{\mathbf{A}}
\end{aligned}
$$






$$
\begin{aligned}
& -\underbrace{\left(\begin{array}{c}
n-1) \\
\widetilde{y}_{i}(0)
\end{array}\right.}_{\mathbf{C}} \\
& -\underbrace{\left.\sum_{\ell=1}^{n-1}\left(\begin{array}{c}
n-1 \\
\ell
\end{array}\right){\stackrel{(\ell)}{\widetilde{x}_{i}}(0)\left(\sum_{j} \gamma_{j i}^{y}{ }^{(n-1-\ell)} \widetilde{y}_{j}(0)\right.}^{(0)}\right)}_{\mathbf{D}} .
\end{aligned}
$$

Recall the assumption

$$
\left\{\begin{array}{l}
y_{k}(0)=\widetilde{y}_{k}(0) \\
x_{k}(0)=\widetilde{x}_{k}(0)
\end{array} \forall k \in \overline{\mathcal{N}}^{(n)}(i) .\right.
$$

From the induction hypothesis, $\forall j \in \mathcal{N}(i), \forall \ell=$ $1, \ldots, n-1, \stackrel{(\ell)}{y_{j}}(0)=\stackrel{(\ell)}{\widetilde{y}_{j}}(0), \stackrel{(\ell)}{x_{j}}(0)=\stackrel{(\ell)}{\widetilde{x}_{j}}(0)$ and also $\stackrel{(\ell)}{y_{i}}(0)=\stackrel{(\ell)}{\widetilde{y}_{i}}(0), \forall \ell \leq n-1$. Therefore, by inspection, we conclude that the terms $\mathbf{A}, \mathbf{B}, \mathbf{C}$, and $\mathbf{D}$ for both equations (39) and (40) match together, and, thus, $\stackrel{(n)}{y_{j}}(0)=\stackrel{(n)}{\widetilde{y}_{j}}(0)$. The same reasoning goes with $\stackrel{(n)}{x_{j}}(0)=\stackrel{(n)}{=} \widetilde{x}_{j}(0)$, and we conclude the proof of the Theorem.

Theorem 24. Let $y_{i}(0)=\widetilde{y}_{i}(0), x_{i}(0)=\widetilde{x}_{i}(0)$ and

$$
\begin{aligned}
& \left\{\begin{array}{l}
y_{k}(0)=\widetilde{y}_{k}(0) \\
x_{k}(0)=\widetilde{x}_{k}(0)
\end{array} \quad \forall k \in \overline{\mathcal{N}}^{n-1}(i)\right. \text { and } \\
& \left\{\begin{array}{l}
y_{k}(0) \geq \widetilde{y}_{k}(0) \\
x_{k}(0) \leq \widetilde{x}_{k}(0)
\end{array} \quad \forall k \in \mathcal{N}^{n}(i) \backslash\{m\}\right.
\end{aligned}
$$

with strict inequality $y_{m}(0)>\widetilde{y}_{m}(0)$ for some $m \in$ $\mathcal{N}^{n}(i)$. Then, $\stackrel{(n)}{y_{i}}(0)>\stackrel{(n)}{\widetilde{y}_{i}}(0)$.

Proof. We apply induction on the number of hops $n$.

Case 1: For $n=1$, from the assumptions of the Theorem, namely, $y_{m}(0)>\widetilde{y}_{m}(0)$ for some $m \in \mathcal{N}(i)$ we conclude that $\dot{y}_{i}(0)>\dot{\widetilde{y}}_{i}(0)$ since

$$
\begin{aligned}
\dot{y}_{i}(0) & =\left(\sum_{j} \gamma_{j i}^{y} y_{j}(0)\right)\left(1-x_{i}(0)-y_{i}(0)\right)-y_{i}(0) \\
& >\left(\sum_{j} \gamma_{j i}^{y} \widetilde{y}_{j}(0)\right)\left(1-x_{i}(0)-y_{i}(0)\right)-y_{i}(0) \\
& =\dot{\tilde{y}}_{i}(0) .
\end{aligned}
$$

Case 2: Induction step. Assume that Theorem 24 holds for $n-1$ and let us prove that it holds for $n$. We consider successively the terms $\mathbf{A}, \mathbf{B}, \mathbf{C}$, and $\mathbf{D}$ in equations (39) and (40).

A: Note that for some $j \in \mathcal{N}(i)$ we have that $m \in \mathcal{N}^{(n-1)}(j)$ where $m$ is defined in the assumptions of the Theorem. Thus, by the induction hypothesis, we have $\stackrel{(n-1)}{y_{j}}(0)>\stackrel{(n-1)}{\widetilde{y}_{j}(0)}$, and, hence, the term A in equation (39) is greater than its counterpart in equation (40).

B and C: From Theorem 23, it should be now clear that these terms match together between equations (39) and (40).

D: From Theorem 23, it follows that $\stackrel{(\ell)}{x_{j}}(0)=\stackrel{(\ell)}{\stackrel{(}{x}_{j}}(0)$ for all $\ell=1, \ldots, n-1$ and thus, term $\mathbf{D}$ is the same for both equations.

Therefore, $\stackrel{(n)}{y}{ }_{j}(0)>\stackrel{(n)}{\widetilde{y}_{j}}(0)$ and the Theorem is proved.

\section{Concluding Remarks}

In this paper, we established a sufficient condition for survival of the fittest in a bi-virus epidemics over bilayer e-networks via a qualitative analysis of the ODE model (4)-(5). More precisely, we have proved that if $G^{y} \preceq^{\star} G^{x}$, then the set

$$
\mathcal{A}=\left[1-\frac{1}{\underline{\gamma}^{x} \underline{d}^{x}}, 1-\frac{1}{\bar{\gamma}^{x} \bar{d}^{x}}\right]^{N} \times\{0\}^{N}
$$

is an attractor of the dynamical system (4)-(5), with basin of attraction given by $\mathcal{B}=\left\{\left(\mathbf{x}_{0}, \mathbf{y}_{0}\right): \mathbf{x}_{0} \neq 0\right\}$, where $G^{y}$ and $G^{x}$ are the e-networks associated with the strains $x$ and $y$. We recall that the inequality ' $\preceq$ ', introduced in this work is defined as

$$
G^{y} \preceq^{\star} G^{x} \Rightarrow G^{y} \preceq \bar{G}^{y} \preceq^{\star} \underline{G}^{x} \preceq G^{x},
$$

where $\underline{G}^{x}=\left(V, \underline{\gamma}^{x}\right), \bar{G}^{y}=\left(V, \bar{\gamma}^{y}\right)$ are inner and outer regular e-networks of $G^{x}$ and $G^{y}$, respectively, and $\bar{G}^{y} \preceq^{\star} \underline{G}^{x}$ means $\bar{\gamma}^{y} \bar{d}^{y} \leq \underline{\gamma}^{x} \underline{d}^{x}$. These concepts allowed us to prove the survival of the fittest sufficient condition without having to resort to Lyapunov stability theory - a Lyapunov function is not known. Namely, we proved that the flow to (4)-(5) of bi-virus epidemics in a general pair of e-networks $G^{x, y}=\left(G^{x}, G^{y}\right)$ preserves skewed bounds on bilayer e-networks: if $S^{x} \preceq G^{x}$, $S^{y} \succeq G^{y}$ then,

$$
\begin{aligned}
& \mathbf{x}\left(t,\left(\mathbf{x}_{0}, \mathbf{y}_{0}\right) ; G^{x, y}\right) \geq \mathbf{x}\left(t,\left(\mathbf{x}_{0}, \mathbf{y}_{0}\right) ; S^{x, y}\right) \\
& \mathbf{y}\left(t,\left(\mathbf{x}_{0}, \mathbf{y}_{0}\right) ; G^{x, y}\right) \leq \mathbf{y}\left(t,\left(\mathbf{x}_{0}, \mathbf{y}_{0}\right) ; S^{x, y}\right) .
\end{aligned}
$$

Then, we combined this result with a qualitative result on regular e-networks (that we proved): if $S^{y} \preceq^{\star} S^{x}$ are regular e-networks, then

$$
\mathbf{y}\left(t,\left(\mathbf{x}_{0}, \mathbf{y}_{0}\right) ; S^{x, y}\right) \longrightarrow \mathbf{0} .
$$

and $\mathbf{x}\left(t,\left(\mathbf{x}_{0}, \mathbf{y}_{0}\right) ; S^{x, y}\right)$ accumulates to the attractor $\mathcal{A}$, if the strain $x$ is strong enough. And that was how we proved the natural selection phenomenon for general enetworks in the main Theorem, Theorem 20. 
The ODE (4)-(5) also admits a non-trivial co-resilient situation: both strains survive in the long run as shown in a numerical simulation depicted in Figure 5. By non-trivial, we mean that the co-resilience takes place for a subset on the rate parameters space of non-zero Lebesgue measure. Figure 5 considers the evolution of a bi-virus epidemics over two e-networks $G^{x}$ and $G^{y}$ where the condition $G^{x} \succeq^{\star} G^{y}$ is violated. In this case, Figure $5 \mathrm{~b}$ shows that the two strains of virus may persist in the network. As future work, we plan to establish

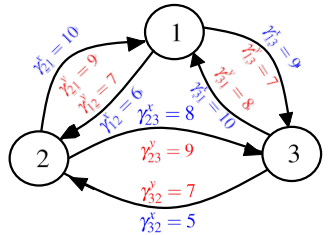

(a)

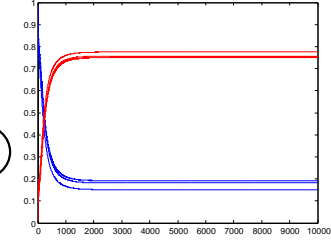

(b)
Figure 5: Epidemics evolution over 3-node e-networks $G^{x}=\left(V, \gamma^{x}\right)$ and $G^{y}=\left(V, \gamma^{y}\right)$. Curves in blue (lighter color) and red (dark color) represent the evolution of the degree of infection at each node by virus strains $x$ and $y$ (one curve per node).

the conditions on the underlying e-networks $G^{x}$ and $G^{y}$ such that there may emerge attractors in the dynamical system (4)-(5) that defy natural selection.

Finally, as we mentioned in the Introduction, the discrete-time counter-part of such logistic ODEs exhibit chaotic behavior and its solutions do not, in general, converge to the equilibria (refer to [11]). Therefore, results on the global stability of such dynamical systems are crucial to understand the behavior of its solutions from the initial conditions to the attractors. Currently, we understand that the overall literature on epidemics, population dynamics, response analysis in bio-chemical chain reactions, food chain or on the study of general logistic dynamical systems over networks is lacking rigorous qualitative analysis. One of the goals of our work is to lay down novel techniques other than Lyapunov stability theory to help close this gap.

\section{REFERENCES}

[1] F. Bauer, "Compartmental models in epidemiology," in Mathematical Epidemiology, F. Bauer, P. Driessche, and J. Wu, Eds. Berlin, Germany: Springer Verlag, 2008, ch. 2, pp. 19-79.

[2] W. H. Hamer, "Epidemic disease in England," The Lancet, vol. 1, pp. 733-9, 1906.

[3] W. O. Kermack and A. G. McKendrick, "A contribution to the mathematical theory of epidemics," Proc. of the Royal Society of London Series A, vol. 115, pp. 700-721, August 1927.

[4] H. W. Hethcote, "A thousand and one epidemic models," in Frontiers in Mathematical Biology, S. A. Levin, Ed. Berlin, Germany: Springer Verlag, 1994, pp. 504-515.

[5] P. Van Mieghem, J. Omic, and R. Kooij, "Virus spread in networks," IEEE/ACM Transactions on Networking,, vol. 17, no. 1, pp. $1-14$, February 2009.
[6] A. Barrat, M. Barthélemy, and A. Vespignani, Dynamical Processes on Complex Networks. London, UK: Cambridge University Press, November 2012.

[7] A. Santos, J. M. F. Moura, and J. M. F. Xavier, "Emergent behavior in multipartite large networks: Multi-virus epidemics," 2013, submitted. http://arxiv.org/abs/1306.6198.

[8] L. C. d. C. Medeiros, C. A. R. Castilho, C. Braga, W. V. de Souza, L. Regis, and A. M. V. Monteiro, "Modeling the Dynamic Transmission of Dengue Fever: Investigating Disease Persistence," PLoS Negl Trop Dis, vol. 5, no. 1, 012011.

[9] X. Wei, N. Valler, B. Prakash, I. Neamtiu, M. Faloutsos, and C. Faloutsos, "Competing memes propagation on networks: A network science perspective," Selected Areas in Communications, IEEE Journal on, vol. 31, no. 6, pp. 1049-1060, June 2013.

[10] M. O. Jackson, Social and Economic Networks. Princeton University Press, 2008.

[11] M. Mitchell, "Complexity: a guided tour," Genetic Programming and Evolvable Machines, vol. 11, no. 1, pp. 127-128, 2010. [Online]. Available: http://dx.doi.org/10.1007/s10710-009-9097-y

[12] A. Lahmanovich and A. Y. James, "A deterministic model for gonorrhea in a nonhomogeneous population," Mathematical Biosciences, vol. 28, pp. 221-236, 1976.

[13] N. Parikh, M. Youssef, S. Swarup, and S. Eubank, "Modeling the effect of transient populations on epidemics in Washington DC," Scientific Reports, vol. 3, no. 3152, 2013.

[14] E. K. Brunson, "The impact of social networks on parents' vaccination decisions," Pediatrics, vol. 131, no. 5, pp. e1397e1404, 2013.

[15] T. Oraby, V. Thampi, and C. T. Bauch, "The influence of social norms on the dynamics of vaccinating behaviour for pediatric infectious diseases," Proc. of the Royal Society B: Biological Sciences, vol. 281, no. 1780, 2014.

[16] O. C. Finney, G. J. Keitany, H. Smithers, A. Kaushansky, S. Kappe, and R. Wang, "Immunization with genetically attenuated p. falciparum parasites induces long-lived antibodies that efficiently block hepatocyte invasion by sporozoites," Vaccine, vol. 32, no. 19, pp. 2135 - 2138, 2014. [Online]. Available: http://www.sciencedirect.com/science/ article/pii/S0264410X14002400

[17] B. Karrer and M. E. J. Newman, "Competing epidemics on complex networks," Phys. Rev. E, vol. 84, p. 036106, Sep 2011. [Online]. Available: http://link.aps.org/doi/10.1103/ PhysRevE.84.036106

[18] C. Darabi Sahneh, Faryad; Scoglio, "May the best meme win!: New exploration of competitive epidemic spreading over arbitrary multi-layer networks," eprint arXiv:1308.4880, August 2013.

[19] B. Bollobas, "The asymptotic number of unlabelled regular graphs," J. of The London Math. Society, pp. 369-382, 1982.

[20] B. McKay and N. Wormald, "Asymptotic enumeration by degree sequence of graphs with degrees $o\left(n^{1 / 2}\right)$," Combinatorica, vol. 11 , no. 4, pp. 369-382, 1991.

[21] B. Bollobas, Random Graphs. Cambridge Univ. Press, 2001.

[22] B. Bollobas, J. Han, and K. J. Verstraete, "Regular subgraphs of random graphs," in Random Structures \& Algorithms, vol. 29, no. 1. Wiley, August 2006, pp. 1-13.

[23] A. Santos and J. M. F. Moura, "Emergent behavior in large scale networks," in 2011 50th IEEE Conference on Decision and Control and European Control Conference (CDC-ECC), December 2011, pp. $4485-4490$. 